\title{
Learning to resist the effects of punishment
}

\author{
WILLIAM TERRIS ${ }^{1}$ AND STANLEY WECHKIN \\ NORTHWESTERN UNIVERSITY MEDICAL SCHOOL
}

In a factorial study albino rats were trained to approach and consume food while receiving either mild shock, mild airblast, or no aversive stimulus and were later tested with either intense shock or airblast. Animals receiving initial training with mild punishment were less affected by subsequent intense aversive stimulation. This decrease in responsiveness to punishment included novel aversive stimuli as well.

Miller (1960) found that rats could be trained to approach and consume food in a runway even though a brief intense electric shock was administered immediately following the beginning of a consummatory response. This was accomplished by initially using a mild electric shock which was then gradually increased until the animals were withstanding intense electric shock. Control animals receiving the same runway training, but without having the experience with gradually increasing shock, stopped approaching the food almost immediately after the full-strength shock was introduced into the runway. This ability to withstand the effects of electric shock could not be attributed to merely experiencing shock since an additional control group, which received a similar series of gradually increasing shocks outside of the runway situation, performed in approximately the same manner as the no-prior-shock control group.

Miller has interpreted his results to indicate "... that rats can be trained to resist stresses such as pain and fear" (Miller, 1960, p. 144). While it is possible that the animals in Miller's study did develop a resistance to fear and pain, the results of his experiment demonstrate only that the animals were able to withstand the effects of electric shock, and are not evidence for a general increase in resistance to fear or pain. If Miller's interpretation is correct, however, animals learning to resist the effects of one aversive stimulus should be able to withstand the effects of novel aversive stimuli as well.

Method

The Ss were 30 male experimentally naive SpragueDawley rats, approximately 95 days of age at the start of the experiment. The animals were housed six per cage with one $S$ from each treatment condition in each group cage. Seven days prior to the start of approach training, all Ss were placed on a $23-\mathrm{hr}$. food deprivation schedule which was maintained throughout the experiment.

Approach training was conducted in a covered runway 36 in. long, 4-1/2 in. high, and 5 in. wide. The floor of the runway was constructed of No. 16 size wire mesh while the last 12 in. of the runway which served as the goal box had a $1 / 8$ in. brass grid floor with the rods spaced $1 / 2$ in. apart. The wooden walls of the runway were painted flat gray and the top consisted of a hinged frame with wire mesh tacked to it. A photocell was attached to the runway so that in the process of placing the animal in the runway an electric clock would be started. A Grason-Stadler Model E6070B shock generator with grid scrambler and an air gun having a source pressure of $28 \mathrm{lb} . / \mathrm{sq}$.in. and an opening of $1 / 8 \mathrm{in}$. in diameter were used to administer the aversive stimuli.

All animals were given a total of 14 approach training trials, one trial each day, in which they were placed in the runway and given a maximum of $2 \mathrm{~min}$. to approach the food and begin eating. Each animal was allowed 30 sec. consummatory time before the trial was terminated. Following this, two Ss from each group cage were randomly assigned to one of three treatment conditions which received on trials 15 through 18 either a mild shock (.25mA for 1 sec.), a mild air blast (a .5 sec. air blast administered approximately 12 in. from the nose of the animal), or no aversive stimulus immediately following the time when the S's nose touched the food. All animals were allowed $2 \mathrm{~min}$. to return to the food after the administration of the aversive stimulus and were allowed $30 \mathrm{sec}$. consummatory time before the trial was terminated. Only one aversive stimulus was administered on each trial.

The intense aversive stimulus was introduced on trial 19 and was continued through trial 24. Each pair of Ss from the group cages trained on weak shock, weak air blast, or no aversive stimulus was randomly divided with one $S$ of each pair receiving strong shock (.5 $\mathrm{mA}$ for $1 \mathrm{sec}$.) and the other receiving a strong air blast (a 1 sec. air blast administered approximately $3 \mathrm{in.}$ from the nose of the animal).

\section{Results and Discussion}

A Kruskal-Wallis nonparametric one way analysis of variance ( $\mathrm{K}-\mathrm{W}$ test) was performed on the time taken to return to the food and begin eating (reconsummatory latency) on trial 15 (the first trial in which the mild aversive stimulus was administered) as a function of the three training conditions (mild shock, mild air blast, or no aversive stimulus) and a significant Chi square was obtained (Chi square $=18.7, \mathrm{df}=$ $2, \mathrm{p}<.01) .^{2}$ The median reconsummatory latencies for the mild shock, mild air blast and no aversive stimulus were $1.3,11.2$, and $0.0 \mathrm{sec}$. respectively. A similar $\mathrm{K}-\mathrm{W}$ test of the consummatory latencies on trial 19 (the last approach trial prior to the actual administration of the intense aversive stimulus)yielded an insignificant Chi square (Chi square $=3.15, \mathrm{df}=2$, 


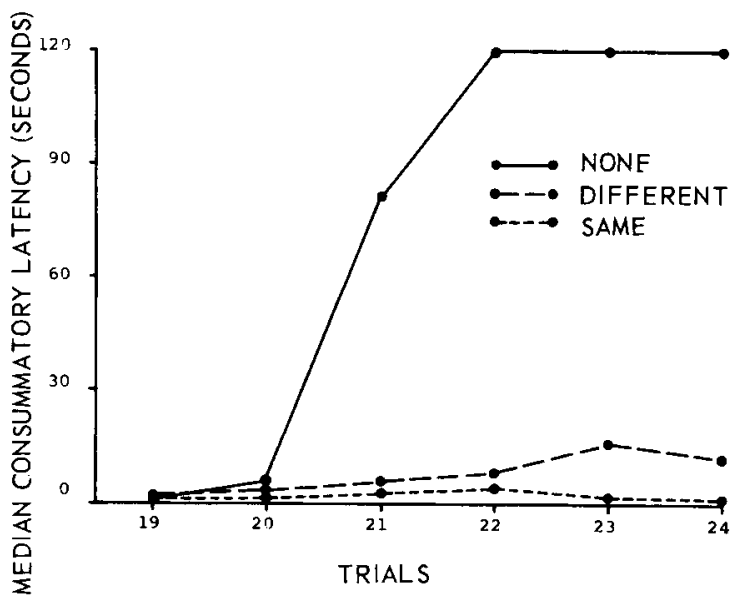

Fig. 1. Median consummatory latencies as a function of the relationship between the initial aversive stimulus encountered in the nuway (mild shock, mild air blast, or no aversive stimulus) and the final test aversive stimulus (strong shock or strong air blast). On trial 19 the intense aversive stimulus is introduced for the first time after the consummatory response has been made.

$p>.20)$. These results indicate that while the mild aversive stimuli had a disrupting effect upon consummatory behavior, all groups entered avoidance testing (trials 19-24) with essentially the same approach strengths.

A two-way analysis of variance was performed on the number of times the Ss approached the food after the introduction of the intense aversive stimulus as a function of the type of aversive stimulus used in avoidance testing (Shock and Air Blast) and the relationship between the initial mild aversive stimulus and the final aversive stimulus used in avoidance testing (Same, Different, and No Prior Aversive Stimulation). Only the relationship $F$ ratio was significant $(F=4.11, \mathrm{df}=$ $2 / 24, p<.05)$. The animals receiving the same aversive stimulus in the training and testing periods had a mean number of approach responses of 4.4 out of a possible 5 (i.e., trials 20-24). Those animals experiencing different aversive stimuli had a mean of 3.6 approach responses, while the animals having no experience with aversive stimulation prior to testing had a mean of 2.3 (F Same vs. None $=8.07, \mathrm{df}=1 / 24$, $p<.01 ;$ F Different vs. None $=3.09, \mathrm{df}=1 / 24, \mathrm{p}<.10$; F Same vs. Different $=1.71, \mathrm{df}=1 / 24, \mathrm{p}>.20$ ).

The median consummatory latencies for the Same, Different, and None groups during avoidance testing are shown in Fig. 1. This data did not meet the assumptions necessary for a parametric analysis since a relatively large number of animals in some groups did not respond on some trials. In order to obtain one score which would represent each animal's performance during avoidance testing, the median con- summatory latency for the five trials following the introduction of the aversive stimulus during avoidance testing was obtained for each $\mathrm{S}$. A K-W test was performed on these consummatory latencies and a significant Chi square was obtained (Chi square $=9.26$, $\mathrm{df}=2, \mathrm{p}<.01$ ). Using Mann-Whitney $U$ tests, it was found that both the consummatory latencies for Same and Different groups were significantly shorter than the None groups (Same vs. None, $\mathrm{p}<.05$; Different vs. None, $p<.05$ ). The Same and Different groups did not differ significantly from each other (Same vs. Different, $p>$.20).

The results of this experiment indicate that rats trained to make a response which results in a mild punishment are better able to withstand the effects of more intense punishment than are animals having no prior experience with the aversive stimulus. This resistance to the effects of punishment includes novel aversive stimuli. While shock and air blast are distinctively different aversive stimuli, they are presumably similar in that they produce a somewhat similar internal state (i.e., fear). If the internal stimuli associated with mild punishment become cues for approach rather than avoidance behavior, more intense punishment would be expected to have less of a disruptive effect. This would be true even if a novel punishment were used. This analysis is consistent with that of Brown \& Wagner (1964) who have noted a similarity between anticipatory frustration and fear and have suggested that resistance to one may result in resistance to the other.

The importance of the context in which the aversive stimulus is experienced is illustrated by the fact that other studies (e.g., Kurtz \& Walters, 1962; Terris \& Wechkin, 1967) have found that electric shock stimulation administered to adult rats in a nonrewarded context typically increases sensitivity to subsequent punishment.

\section{References}

Brown, R. T., \& Wagner, A. Resistance to punishment and extinction following training with shock or nonreinforcement. $J$. exp. Psychol., 1964, 68, 503-507.

Kurtz, K., \& Walters, G. The effects of prior fear experiences on an approach-avoidance conflict. J. comp. physiol. Psychol., $1962,55,1075-1078$.

Miller, N. E. Learning resistance to pain and fear: effects of overlearning, exposure and rewarded exposure in context. $J$. exp. Psychol., 1961, 60, 137-145.

Terris, W., \& Wechkin, S. Approach-avoidance conflict behavior as a function of prior experiences with mild or intense electric shock stimulation. Psychon. Sci., 1967, 7, 39-40.

\section{Notes}

1. Present address: Department of Psychology, University of Oklahoma, Norman, Oklahoma.

2. All probability statements are based upon two-tailed tests. 